\title{
The endocannabinoid system: a key modulator of emotions and cognition
}

\author{
Patrizia Campolongo $^{1}$ and Viviana Trezza ${ }^{2 *}$ \\ ${ }^{1}$ Department of Physiology and Pharmacology, University of Rome Sapienza, Rome, Italy \\ 2 Department of Biology, University "Roma Tre," Rome, Italy \\ ${ }^{*}$ Correspondence: viviana.trezza@uniroma3.it \\ Edited by: \\ Carmen Sandi, École Polytechnique Fédérale de Lausanne, Switzerland
}

The endocannabinoid system is a unique neuromodulatory system in mammalian physiology. It consists of cannabinoid receptors ( $\mathrm{CB} 1$ and $\mathrm{CB} 2$ ), their endogenous lipid ligands [endocannabinoids, including anandamide (AEA), and 2-arachidonoylglycerol (2-AG)] and the enzymes for ligand synthesis and degradation. In recent years, brain endocannabinoids have emerged as key modulators of affect, motivation and emotions, and the endocannabinoid system is nowadays considered an intriguing target for the development of selective and specific compounds able to treat several psychiatric disorders. This e-book brings together leading experts in the field to provide a deep overview of the physiological and pathophysiological role of the endocannabinoid system in the modulation of emotions and cognition.

The e-book opens with a review where Battista et al. provide a general overview on the endocannabinoid system and then focus on the metabolic and signal transduction pathways of the main endocannabinoids, AEA and 2-AG. At the end, the authors briefly discuss the therapeutic potential of new cannabinoid drugs (Battista et al., 2012). This issue is further elaborated in the following review, where Marco et al. provide both clinical and preclinical evidence supporting the involvement of the endocannabinoid system in several neuropsychiatric disorders (Marco et al., 2011).

The role of the endocannabinoid system in the modulation of emotions and cognition is widely underscored by several reviews of this e-book. Zanettini et al. broadly introduce this topic by discussing the results of studies performed in laboratory animals (Zanettini et al., 2011), while Rubino and Parolaro address this issue from a sexually-dimorphic perspective (Rubino and Parolaro, 2011).

The original research article by Terzian et al. investigated the potential cross-talk between dopaminergic and cannabinoid neurotransmission in the modulation of emotions and cognition (Terzian et al., 2011). The authors showed that conditional CB1 receptor knock-out animals lacking $\mathrm{CB} 1$ cannabinoid receptors in neurons expressing D1dopamine receptors exhibited significantly increased contextual and auditory-cued fear compared to wild-type animals, suggesting that a specific reduction of endocannabinoid signaling in neurons expressing dopamine D1 receptor is able to affect acute fear adaptation (Terzian et al., 2011). In their commentary on this research article, Akirav and Fattore discuss about the potential clinical implication of these findings, and indicate the future directions for research in this field (Akirav and Fattore, 2011).

The preclinical studies reviewed by Trezza and co-workers show that cannabinoid modulation of emotionality and cognitive performance appears since early developmental stages; indeed, evidence has been provided over the last few years that animals exposed to cannabinoid drugs during the perinatal, prenatal or adolescent period show long-lasting changes in emotional reactivity and cognitive processing (Campolongo et al., 2007, 2009, 2011; Trezza et al., 2012).

The effects of cannabinoid drugs on hippocampal memory and plasticity are discussed by Akirav (Akirav, 2011); on the basis of the existing literature, she concludes that these effects may vary depending on the route of drug administration, the nature of the task used, whether it involves emotional or nonemotional memory formation, and according to the memory stage under investigation (acquisition, consolidation, retrieval, and extinction) and the brain areas involved (Akirav, 2011).

To study the role of CB1 cannabinoid receptors in the medial prefrontal cortex on cognitive flexibility and emotional behavior in rats, Klugmann et al. upregulated CB1 cannabinoid receptors selectively in this brain area by using adeno-associated viral vector-mediated gene transfer (Klugmann et al., 2011). In their research article, these authors showed that upregulation of $\mathrm{CB} 1$ receptors specifically in the rat medial prefrontal cortex induces alterations in emotional reactivity, leads to inadequate social behavior, and impairs cognitive flexibility (Klugmann et al., 2011). In the following research article published on this e-book, Hernandez et al. shed more light on the role of CB1 cannabinoid receptors in mediating reward-seeking behaviors (Hernandez et al., 2011). In particular, the authors showed that, unlike lithium chloride, the CB1 receptor antagonist AM251 did not affect instrumental responding for brain stimulation reward. On the basis of these findings, the authors hypothesize that endocannabinoids are primarily involved with the motivational rather than the intrinsic aspects of reward processing (Hernandez et al., 2011).

The last three articles included in this e-book address the topic of cannabinoid modulation of emotions and cognition from a clinical perspective. The first of these studies is a research article where Spronk and colleagues showed that the active ingredient of Cannabis $\Delta$ 9-tetrahydrocannabinol (THC) alters performance monitoring, that is a process that allows humans to respond actively and safely to changing environmental demands 
(Spronk et al., 2011). This study supports the opinion that Cannabis use during performance of complex functions like driving, which require a high level of performance monitoring, might be particularly risky. Fattore and Fratta address a very hot and timely topic, that is the availability of a new generation of drugs that, although devoid of tobacco or Cannabis, when smoked produce effects similar to those induced by THC (Fattore and Fratta, 2011). The authors first outline the general characteristics of these drugs, such as their content and their effects, and then address the consequences that their use has for both health and society (Fattore and Fratta, 2011). The last contribution to this e-book is the opinion article by Bhattacharyya and Sendt,

\section{REFERENCES}

Akirav, I. (2011). The role of cannabinoids in modulating emotional and non-emotional memory processes in the hippocampus. Front. Behav. Neurosci. 5:34. doi: 10.3389/fnbeh.2011.00034

Akirav, I., and Fattore, L. (2011). Cannabinoid CB1 and dopamine D1 receptors partnership in the modulation of emotional neural processing. Front. Behav. Neurosci. 5:67. doi: 10.3389/fnbeh.2011.00067

Battista, N., Di Tommaso, M., Bari, M., and Maccarrone, M. (2012). The endocannabinoid system: an overview. Front. Behav. Neurosci. 6:9. doi: 10.3389/ fnbeh.2012.00009

Bhattacharyya, S., and Sendt, K. V. (2012). Neuroimaging evidence for cannabinoid modulation of cognition and affect in man. Front. Behav. Neurosci. 6:22. doi: 10.3389/fnbeh.2012.00022

Campolongo, P., Trezza, V., Cassano, T., Gaetani, S., Morgese, M. G., Ubaldi, M., et al. (2007). Perinatal exposure to delta-9-tetrahydrocannabinol causes enduring cognitive deficits associated with alteration of cortical gene expression and neurotransmission in rats. Addict. Biol. 12, 485-495.
Campolongo, P., Trezza, V., Palmery, M., Trabace, L., and Cuomo, V. (2009). Developmental exposure to cannabinoids causes subtle and enduring neurofunctional alterations. Int. Rev. Neurobiol. 85, 117-133.

Campolongo, P., Trezza, V., Ratano, P., Palmery, M., and Cuomo, V. (2011). Developmental consequences of perinatal cannabis exposure: behavioral and neuroendocrine effects in adult rodents. Psychopharmacology (Berl.) 214, 5-15.

Fattore, L., and Fratta, W. (2011). Beyond THC: the new generation of cannabinoid designer drugs. Front. Behav. Neurosci. 5:60. doi: 10.3389/fnbeh.2011.00060

Hernandez, G., Bernstein, D., Schoenbaum, G., and Cheer, J. F. (2011). Contrasting effects of lithium chloride and CB1 receptor blockade on enduring changes in the valuation of reward. Front. Behav. Neurosci. 5:53. doi: 10.3389/fnbeh.2011.00053

Klugmann, M., Goepfrich, A., Friemel, C. M., and Schneider, M. (2011). AAV-Mediated overexpression of the $\mathrm{CB} 1$ receptor in the $\mathrm{mPFC}$ of adult rats alters cognitive flexibility, social behavior, and emotional reactivity. Front. Behav. Neurosci. 5:37. doi: $10.3389 /$ fnbeh.2011.00037

that provides evidence from neuroimaging studies that cannabinoid drugs affect brain areas involved in cognitive and emotional processes (Bhattacharyya and Sendt, 2012).

Altogether, the collection of articles included in this e-book demonstrates that endocannabinoids play a crucial role in the regulation of emotionality and cognitive performance, as outlined by both rodent and human studies. We hope that it will be apparent to the readers how far we have come in recent years in understanding the functions of brain endocannabinoids in both physiological and pathological conditions, and which are the current challenges for researchers working in this field.

Marco, E. M., Garcia-Gutierrez, M. S. Bermudez-Silva, F. J., Moreira, F. A., Guimaraes, F., Manzanares, J., et al. (2011). Endocannabinoid system and psychiatry: in search of a neurobiological basis for detrimental and potential therapeutic effects. Front. Behav. Neurosci. 5:63. doi: 10.3389/fnbeh.2011.00063

Rubino, T., and Parolaro, D. (2011) Sexually dimorphic effects of cannabinoid compounds on emotion and cognition. Front. Behav. Neurosci. 5:64. doi: 10.3389/fnbeh.2011.00064

Spronk, D., Dumont, G. J. Verkes, R. J., and De Bruijn, E. R. (2011). Acute effects of delta-9-tetrahydrocannabinol on performance monitoring in healthy volunteers. Front. Behav. Neurosci. 5:59. doi: 10.3389/fnbeh.2011.00059

Terzian, A. L., Drago, F., Wotjak, C. T., and Micale, V. (2011) The dopamine and cannabinoid interaction in the modulation of emotions and cognition: assessing the role of cannabinoid CB1 receptor in neurons expressing dopamine D1 receptors. Front. Behav. Neurosci. 5:49. doi: 10.3389/fnbeh.2011.00049

Trezza, V., Campolongo, P., Manduca, A., Morena, M., Palmery, M.,
Vanderschuren, L. J., et al. (2012). Altering endocannabinoid neurotransmission at critical developmental ages: impact on rodent emotionality and cognitive performance. Front. Behav. Neurosci. 6:2. doi: 10.3389/ fnbeh.2012.00002

Zanettini, C., Panlilio, L. V., Alicki, M., Goldberg, S. R., Haller, J., and Yasar, S. (2011). Effects of endocannabinoid system modulation on cognitive and emotional behavior. Front. Behav. Neurosci. 5:57. doi: 10.3389/fnbeh.2011.00057

Received: 18 October 2012; accepted: 18 October 2012; published online: 06 November 2012.

Citation: Campolongo $P$ and Trezza V (2012) The endocannabinoid system: $a$ key modulator of emotions and cognition. Front. Behav. Neurosci. 6:73. doi: 10.3389/fnbeh.2012.00073

Copyright (c) 2012 Campolongo and Trezza. This is an open-access article distributed under the terms of the Creative Commons Attribution License, which permits use, distribution and reproduction in other forums, provided the original authors and source are credited and subject to any copyright notices concerning any third-party graphics etc. 